\title{
OPTIMAL LIQUIDATION OF A PAIRS TRADE
}

\author{
ERIK EKSTRÖM ${ }^{1}$, CARL LINDBERG, JOHAN TYSK ${ }^{1}$
}

\begin{abstract}
Pairs trading is a common strategy used by hedge funds. When the spread between two highly correlated assets is observed to deviate from historical observations, a long position is taken in the underpriced asset and a short position in the overpriced one. If the spread narrows, both positions are closed, thus generating a profit. We study when to optimally liquidate a pairs trading strategy when the difference between the two assets is modeled by an Ornstein-Uhlenbeck process. We also provide a sensitivity analysis in the model parameters.
\end{abstract}

\section{INTRODUCTION}

Consider a pair of assets having price processes with a difference fluctuating about a given level. A typical example is stocks of two companies in the same area of business. If the spread between the two price processes at some point widens, then one of the assets is underpriced relative to the other one. An investor wanting to benefit from this relative mis-pricing may invest in a pairs trade, i.e. the investor buys the (relatively) underpriced asset and takes a short position in the (relatively) overpriced one. When the spread narrows again, the position is liquidated and a profit is made. Note that the holder of a pairs trade is not exposed to market risk but instead tries to benefit from relative price movements, thus making pairs trade a common hedge fund strategy.

The literature on trading strategies used by hedge funds seems to be somewhat limited compared to its practical significance. However, there are a number of recent books that treat the applied aspects of pairs trading, see [1], [5] and [6]; for a historical evaluation of pairs trading, see also [3]. The authors of [2] model pair spreads as mean reverting Gaussian Markov chains observed in Gaussian noise. Our approach is the continuous time analogue of this since we use mean-reverting Ornstein-Uhlenbeck processes to model the spread. We thus model the difference $X$ between the two assets as

$$
d X_{t}=-\mu X_{t} d t+\sigma d W_{t},
$$

where $\mu$ and $\sigma$ are positive constants and $W$ is a standard Brownian motion.

2000 Mathematics Subject Classification. Primary 91B28; Secondary 60G40.

Key words and phrases. Pairs trading; Optimal stopping problem; Ornstein-Uhlenbeck process.

${ }^{1}$ Support from the Swedish Research Council is gratefully acknowledged. 
Note that there is a large model risk associated to the pairs trading strategy. Indeed, if it turns out that the difference between the assets is no longer mean-reverting, then the investor faces a considerable risk. What is typically done in practice is that the investor decides (in advance) on a stop-loss level $B<0$, and if the value of the pair trade falls below $B$ then one liquidates the position and accepts the loss. The stop-loss level $B$ can be seen as a (crude) model adjustment: if this level is reached then the model is abandoned and the position is closed. A natural continuation of our work would be to introduce a continuous recalibration of the model parameters to decrease the model risk.

In Section 2, we formulate and solve explicitly the optimal stopping problem of when to liquidate a pair trade in the presence of a stop-loss barrier. In Section 3 we study the dependence of the optimal liquidation level on the different model parameters, thus providing a better understanding of the consequences of possible mis-specifications of the model. More precisely, we show that increasing the quotient $\alpha=2 \mu / \sigma^{2}$ increases the optimal liquidation level, and that the optimal liquidation level is between $-B / 2$ and $-B$ for any choice of parameters $\mu$ and $\sigma$. In Section 4 we consider the optimal liquidation of a pairs trade in the presence of a discount factor. When including such a discount factor, the dependence on the model parameters becomes more delicate, and a numerical study is conducted. Finally, we also consider the optimal liquidation problem in the absence of a stop-loss barrier.

\section{Solving the optimal STOPPING PROBlem}

If we assume that any fraction of an asset can be traded, then there is no loss of generality to assume that the difference between the two assets fluctuates about the level 0 . As explained in the introduction, we model the difference $X$ between the two assets as a mean-reverting Ornstein-Uhlenbeck process, i.e.

$$
d X_{t}=-\mu X_{t} d t+\sigma d W_{t}
$$

Here $\mu$ and $\sigma$ are positive constants and $W$ is a standard Brownian motion. For a given liquidation level $B<0$, define the value $V$ of the option spread by

$$
V(x)=\sup _{\tau \leq \tau_{B}} E_{x} X_{\tau},
$$

where the supremum is taken over all stopping times that are smaller than

$$
\tau_{B}=\inf \left\{t: X_{t} \leq B\right\},
$$

the first hitting time of the liquidation level $B$. The stop-loss level $B$ is imposed to have a bound on the possible losses. Of course, if the model (1) is known to be true, then the spread would vanish eventually since $X$ has a mean-reverting drift. However, in practice a stop-loss level has to 
be imposed to account for the risk that the model is incorrect. The stoploss level $B$ thus makes the risk involved in pairs trading less sensitive to a possible mis-specification of the model.

If the process $X$ is negative, then the drift is positive so one should not liquidate the position. If $X$ is positive then the negative drift works against the owner of the pair. For large values of $X$, this drift is substantial and should outweigh the possible benefits of the random fluctuations. This indicates that there exists a boundary $x=b$ above which liquidation is optimal, and below which the pair should be kept.

General optimal stopping theory then suggests that the pair $(V, b)$ solves

$$
\left\{\begin{array}{l}
\frac{\sigma^{2}}{2} V_{x x}-\mu x V_{x}=0 \quad \text { if } x \in(B, b) \\
V(B)=B \\
V(b)=b \\
V^{\prime}(b)=1 .
\end{array}\right.
$$

The general solution to the ordinary differential equation $\frac{\sigma^{2}}{2} V_{x x}-\mu x V_{x}=0$ is

$$
V(x)=C F(x)+D .
$$

Here $C$ and $D$ are constants,

$$
\begin{gathered}
F(x)=\int_{0}^{x} f(y) d y \\
f(y)=e^{\alpha y^{2} / 2}
\end{gathered}
$$

and $\alpha=2 \mu / \sigma^{2}$ is the reciprocal of the variance of the stationary distribution of $X$. Inserting the general solution into the free boundary problem, the equation

$$
\frac{F(b)-F(B)}{b-B}=f(b)
$$

for the exercise boundary $b$ is derived.

Lemma 2.1. Equation (4) admits a unique solution b larger than B. Moreover, $b \in(0,-B)$.

Proof. Define

$$
g(x):=F(x)-F(B)-(x-B) f(x),
$$

and note that $b>B$ is a solution of (4) if and only if $g(b)=0$. We have $g(B)=0, g^{\prime}(x)=-\alpha(x-B) x f(x) \geq 0$ if $x \in[B, 0]$ and $g^{\prime}(x)<0$ if $x>0$. Moreover,

$$
g(-B)=-2 F(B)+2 B f(B)<0
$$

since $F$ is convex. Consequently, $g$ has a unique zero $x=b$ larger than $B$, and $b \in(0,-B)$. 
Now, given the unique solution $b$ of equation (4), let

$$
\hat{V}(x)= \begin{cases}\frac{F(x)}{f(b)}+B-\frac{F(B)}{f(b)} & B \leq x<b \\ x & x \geq b\end{cases}
$$

It is easy to check that $(\hat{V}, b)$ is the unique solution to the free boundary problem (3). Moreover, it follows from the proof of Lemma 2.1 above that $\hat{V}(x) \geq x$ for all $x \geq B$.

Theorem 2.2. The value function $V$ coincides with the function $\hat{V}$ given in (6). Moreover, $\tau^{*}=\tau_{B} \wedge \tau_{b}$ is an optimal stopping time in (2).

Proof. Consider the process $Y_{t}=\hat{V}\left(X_{t \wedge \tau_{B}}\right)$. By (a generalised version of) Ito's lemma,

$$
\begin{aligned}
Y_{t}= & \hat{V}(x)+\int_{0}^{t \wedge \tau_{B}}\left(\frac{\sigma^{2}}{2} \hat{V}_{x x}\left(X_{s}\right)-\mu X_{s} \hat{V}_{x}\left(X_{s}\right)\right) I\left(X_{s} \neq b\right) d s \\
& +\int_{0}^{t \wedge \tau_{B}} \sigma \hat{V}_{x}\left(X_{s}\right) I\left(X_{s} \neq b\right) d W \\
= & \hat{V}(x)-\mu \int_{0}^{t \wedge \tau_{B}} X_{s} I\left(X_{s}>b\right) d s+\int_{0}^{t \wedge \tau_{B}} \sigma \hat{V}_{x}\left(X_{s}\right) d W .
\end{aligned}
$$

The Ito integral is a martingale since the integrand is bounded. Therefore, since $b$ is positive, the process $Y$ is a supermartingale. If $\tau$ is a stopping time, then the optional sampling theorem, see Problem 3.16 and Theorem 3.22 in [4], gives that

$$
E X_{\tau \wedge \tau_{B}} \leq E \hat{V}\left(X_{\tau \wedge \tau_{B}}\right)=E Y_{\tau} \leq E Y_{0}=\hat{V}(x) .
$$

Since $\tau$ is arbitrary, this yields

$$
V(x) \leq \hat{V}(x) .
$$

To derive the reverse inequality, note that $Y_{t \wedge \tau_{b}}$ is a bounded martingale, and that $Y_{\tau_{b}}=X_{\tau_{b} \wedge \tau_{B}}$. It follows that the inequalities in $(7)$ reduce to equalities if $\tau=\tau^{*}$, which finishes the proof.

\section{Dependence on parameters}

It is easy to see that the value $V$ and the optimal threshold $b$ are both increasing as functions of the absolute value $|B|$ of the stop-loss level. Indeed, this follows since a large $|B|$ increases the set of stopping times smaller than $\tau_{B}$. The dependence on the parameters $\mu$ and $\sigma$ is more delicate, and given by the theorem below.

Theorem 3.1. The optimal stopping boundary $b$ is increasing as a function of $\alpha=2 \mu / \sigma^{2}$, and it satisfies $\lim _{\alpha \downarrow 0} b(\alpha)=-B / 2$ and $\lim _{\alpha \uparrow \infty} b(\alpha)=-B$.

Proof. Define

$$
g(x, \alpha):=\int_{B}^{x} e^{\alpha y^{2} / 2} d y-(x-B) e^{\alpha x^{2} / 2}
$$


compare (5). Recall that for a fixed $\alpha>0$, the function $x \mapsto g(x, \alpha)$ satisfies $g(0, \alpha)>0, g(-B, \alpha)<0$ and $\frac{\partial g}{\partial x}(x, \alpha)<0$ for $x>0$. Moreover, the optimal stopping boundary $b \in(0,-B)$ is the unique positive value such that $g(b, \alpha)=0$. Let $x \in(0,-B)$. Expanding into Taylor series, we have

$$
\begin{aligned}
\int_{B}^{x} e^{\alpha y^{2} / 2} d y & =\int_{B}^{x} \sum_{k=0}^{\infty} \frac{(\alpha / 2)^{k}}{k !} y^{2 k} d y \\
& =\sum_{k=0}^{\infty} \frac{(\alpha / 2)^{k}}{k !(2 k+1)}\left(x^{2 k+1}-B^{2 k+1}\right) \\
& =\sum_{k=0}^{\infty} \frac{(\alpha / 2)^{k}}{k !(2 k+1)} x^{2 k}(x-B) a_{k},
\end{aligned}
$$

where

$$
a_{k}=1+(B / x)+\ldots+(B / x)^{2 k} .
$$

Using

$$
e^{\alpha x^{2} / 2}=\sum_{k=0}^{\infty} \frac{(\alpha / 2)^{k} x^{2 k}}{k !}
$$

we find that

$$
g(x, \alpha)=(x-B) \sum_{k=1}^{\infty} \frac{(\alpha / 2)^{k} x^{2 k}}{k !}\left(\frac{a_{k}}{2 k+1}-1\right) .
$$

Note that there exists $k_{0}$ such that $a_{k} \leq 2 k+1$ for $k \leq k_{0}$ and $a_{k}>2 k+1$ for $k>k_{0}$. Let $b$ be the unique zero of $g(x, \alpha)$ for a given $\alpha$. Now, if $\alpha^{\prime}>\alpha$, then

$$
\begin{aligned}
0 & =g(b, \alpha)=(x-B)(\alpha / 2)^{k_{0}} \sum_{k=1}^{\infty} \frac{(\alpha / 2)^{k-k_{0}} x^{2 k}}{k !}\left(\frac{a_{k}}{2 k+1}-1\right) \\
& <(x-B)(\alpha / 2)^{k_{0}} \sum_{k=1}^{k_{0}} \frac{\left(\alpha^{\prime} / 2\right)^{k-k_{0}} x^{2 k}}{k !}\left(\frac{a_{k}}{2 k+1}-1\right) \leq g\left(b, \alpha^{\prime}\right) .
\end{aligned}
$$

It follows that $g\left(b, \alpha^{\prime}\right)>0$, so the unique zero $x=b^{\prime}$ of $g\left(x, \alpha^{\prime}\right)$ satisfies $b^{\prime} \geq$ $b$, which proves the claimed monotonicity of the optimal stopping boundary $b$ as a function of $\alpha$.

Finally we consider the limits in the statement of the theorem, starting with $\alpha$ tending to infinity. Recall from above that $b(\alpha)<-B$. On the other hand, for a fixed $\epsilon>0$ we have

$$
g(-B-\varepsilon, \alpha)>0
$$

for $\alpha$ large enough since the integral term in the definition of $g$ is bounded below by $\frac{\epsilon}{2} e^{\alpha(-B-\epsilon / 2)^{2} / 2}$, whereas the remaining term is $O\left(e^{\alpha(-B-\epsilon)^{2} / 2}\right)$. Hence $b(\alpha)>-B-\epsilon$ and the desired conclusion follows. Next we consider the limit as $\alpha$ tends to zero. The argument here is based on the approximation of 
$e^{x}$ by $1+x$ for small $x$. Thus we replace the exponential functions in the definition av $g$ by $1+\alpha y^{2} / 2$ and $1+\alpha x^{2} / 2$, and define

$$
\begin{aligned}
h(x, \alpha) & :=\int_{B}^{x}\left(1+\alpha y^{2} / 2\right) d y-(x-B)\left(1+\alpha x^{2} / 2\right) \\
& =\frac{\alpha}{6}\left(3 B x^{2}-2 x^{3}-B^{3}\right) .
\end{aligned}
$$

One finds that $h(-B / 2, \alpha)=0$ for all $\alpha$. The derivative $\frac{\partial h}{\partial x}(x, \alpha)=\alpha(B x-$ $x^{2}$ ) is negative and is bounded above and below by positive multiples of $\alpha$ in a neighborhood of $-B / 2$. Since the error in the approximation of the exponential function with the linear function is of order $\alpha^{2}$, the result follows.

Remark In the absence of a stop-loss level (and a discount factor), i.e. if $B=-\infty$ in the above set-up, it follows from Theorem 3.1 that the optimal liquidation level $b=\infty$. Thus the problem degenerates and it is never optimal to liquidate the pairs trade.

\section{INCLUDING A DISCOUNT FACTOR}

It may be of interest to include a discounting factor in the analysis above, thus instead considering the optimal stopping problem

$$
V(x)=\sup _{\tau \leq \tau_{B}} E_{x} e^{-r \tau} X_{\tau},
$$

where $r>0$ is a constant. This optimal stopping problem can, in principle, be studied using similar techniques as in the problem with no discounting. However, it turns out that the solution is slightly less explicit, and the parameter dependences are more involved.

Again, it is natural to expect that the optimal stopping time takes the form of the first hitting time of a level $b$. The same arguments as in Section 2 suggest that the pair $(V, b)$ solves

$$
\left\{\begin{array}{l}
\frac{\sigma^{2}}{2} V_{x x}-\mu x V_{x}-r V=0 \quad \text { if } x \in(B, b) \\
V(B)=B \\
V(b)=b \\
V^{\prime}(b)=1 .
\end{array}\right.
$$

The general solution to the ordinary differential equation $\frac{\sigma^{2}}{2} V_{x x}-\mu x V_{x}-$ $r V=0$ is

$$
V(x)=C F(x)+D G(x) .
$$

Here $C$ and $D$ are constants and

$$
\begin{gathered}
F(x)=\int_{0}^{\infty} u^{\beta-1} e^{\sqrt{\alpha} x u-u^{2} / 2} d u, \\
G(x)=F(-x)
\end{gathered}
$$


and $\alpha=2 \mu / \sigma^{2}$ and $\beta=r / \mu$. Inserting the general solution into the free boundary problem, it is easily seen that

$$
C=\frac{B G(b)-b G(B)}{G(b) F(B)-G(B) F(b)}
$$

and

$$
D=\frac{b F(B)-B F(b)}{G(b) F(B)-G(B) F(b)},
$$

where $b$ satisfies

$$
(B G(b)-b G(B)) F^{\prime}(b)+(b F(B)-B F(b)) G^{\prime}(b)=G(b) F(B)-G(B) F(b) .
$$

Arguing as in the proof of Theorem 2.2, it is straightforward to check that the value function derived above coincides with the value of the optimal stopping problem (8).

Theorem 4.1. Let $b$ be the unique solution of $(12)$ in $(0,-B)$, and define $C$ and $D$ as in (10) and (11), respectively. The value function of the optimal stopping problem (8) is given by

$$
V(x)= \begin{cases}C F(x)+D G(x) & x \in(B, b) \\ x & x \geq b .\end{cases}
$$

Moreover, $\tau^{*}=\tau_{B} \wedge \tau_{b}$ is an optimal stopping time in (8).

In the absence of a stop-loss level, i.e. if $B=-\infty$, then

$$
V(x)= \begin{cases}\frac{b}{F(b)} F(x) & x<b \\ x & x \geq b,\end{cases}
$$

where $b$ is the unique positive solution of $F(b)=b F^{\prime}(b)$.

Proof. The proof of the optimality follows along the same lines as in the proof of Theorem 2.2, and we omit the details. To prove the uniqueness of solutions to (12), define

$$
\begin{aligned}
f(x)= & (B G(x)-x G(B)) F^{\prime}(x)+(x F(B)-B F(x)) G^{\prime}(x) \\
& +G(B) F(x)-G(x) F(B) .
\end{aligned}
$$

Then $f(b)=0$ if and only if $b$ solves equation (12). First note that

$$
f(0)=F(0)\left(2 B F^{\prime}(0)+F(-B)-F(B)\right) .
$$

Since

$$
\begin{aligned}
F(-B)-F(B) & =\int_{0}^{\infty} u^{\beta-1}\left(e^{-\sqrt{\alpha} B u}-e^{\sqrt{\alpha} B u}\right) e^{-u^{2} / 2} d u \\
& >-\int_{0}^{\infty} u^{\beta} 2 \sqrt{\alpha} B e^{-u^{2} / 2} d u=-2 B F^{\prime}(0),
\end{aligned}
$$

we find that $f(0)>0$. Similarly,

$$
f(-B)=(F(B)+F(-B))\left(B F^{\prime}(B)+B F^{\prime}(-B)+F(-B)-F(B)\right) .
$$


It is easy to check that $g(x):=-x F^{\prime}(-x)-x F^{\prime}(x)+F(x)-F(-x)$ satisfies $g(0)=0$ and $g^{\prime}(x)<0$ for $x>0$. Consequently, $f(-B)<0$, so there exists a zero of $f$ in the interval $(0,-B)$. Moreover,

$$
f^{\prime}(x)=(B F(-x)-x F(-B)) F^{\prime \prime}(x)+(x F(B)-B F(x)) F^{\prime \prime}(-x) .
$$

Since $F^{\prime \prime}(x)>F^{\prime \prime}(-x)$ and $x F(B)-B F(x)>B F(-x)-x F(-B)$ for $x \in(0,-B)$, we have $f^{\prime}(x)<0$ in that interval. Thus the function $f$ has a unique zero in $(0,-B)$, so there exists a unique solution $b$ to (12).

Remark As indicated above, the parameter dependencies are more involved in the presence of a discount factor, compare Figure 1. If the stop-loss level satisfies $B=-\infty$, however, then it is straightforward to check that the optimal liquidation level $b$ is decreasing in the parameter $\alpha$ and in the parameter $\beta$.

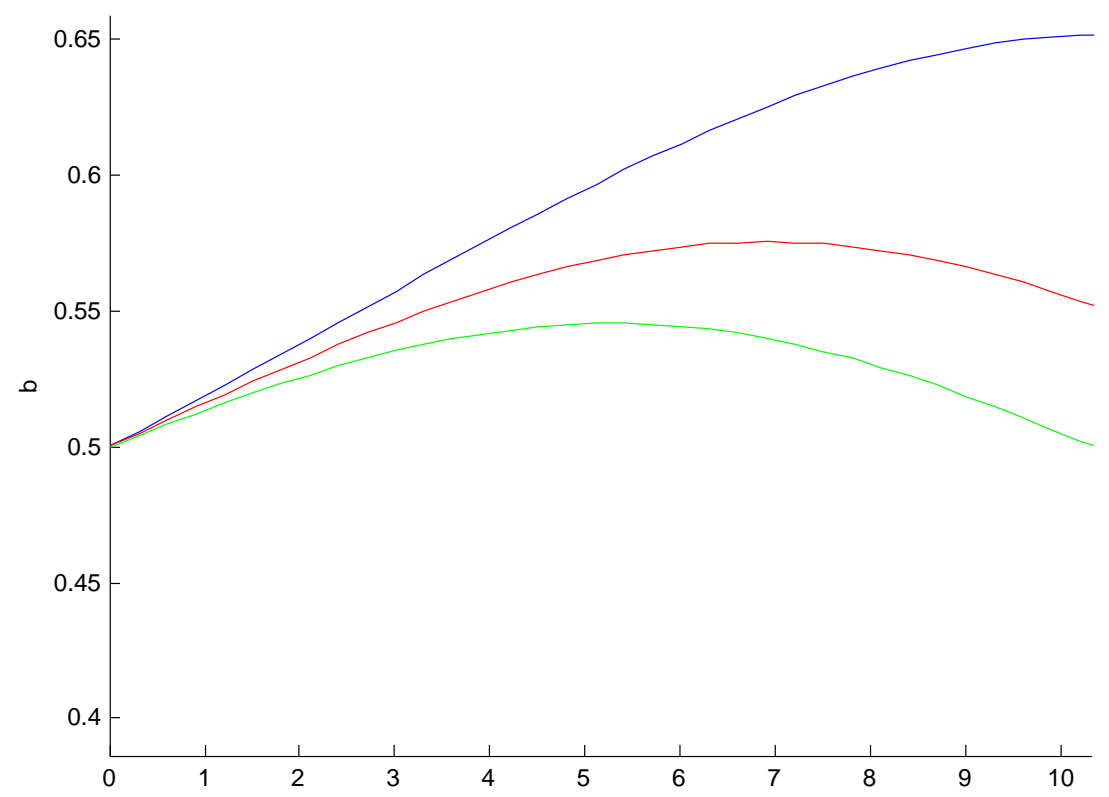

FigURE 1. The graph shows the optimal threshold $b$ as a function of the parameter $\alpha$ for three different values of $\beta$. The values of $\beta$ are 0.01 (top), 0.05 (middle) and 0.09 (bottom). In all three examples we used $B=-1$.

\section{REFERENCES}

[1] Ehrman, D. The Handbook of Pairs Trading. John Wiley \& Sons Inc, 2006.

[2] Elliott, R., van der Hoek, J., and Malcolm, W. Pairs trading. Quantitative Finance 5 (2005), 271-276.

[3] Gatev, E., Goetzmann, W. and Rouwenhorst, G. Pairs trading: performance of a relative-value arbitrage, Review of Financial Studies 19, 797-827. 
[4] Karatzas, I. and Shreve, S. Brownian Motion and Stochastic Calculus. 2nd ed., Springer-Verlag, New York, 2000.

[5] Vidyamurthy, G. Pairs Trading - Quantitative Methods and Analysis, John Wiley \& Sons Inc, New Jersey, 2004.

[6] Whistler, M. Trading Pairs: Capturing Profits and Hedging Risk with Statistical Arbitrage Strategies, John Wiley \& Sons Inc, 2004.

E. Ekström and J. Tysk: Department of Mathematics, Uppsala University, Box 480, SE-751 06 UpPSALA, SWEDEN

C. Lindberg: Mathematical Sciences, Chalmers University of Technology, 41296 GÖTEBOrG, SWEDEN 